\title{
A PSCAD Processor-in-the-loop System For Hardware Evaluation of Power Converter Control Algorithms
}

DOI:

10.1049/icp.2021.1060

Link to publication record in Manchester Research Explorer

\section{Citation for published version (APA):}

Andrews, J., Green, P., \& Barnes, M. (2020). A PSCAD Processor-in-the-loop System For Hardware Evaluation of Power Converter Control Algorithms. In The 10th International Conference on Power Electronics, Machines and Drives (PEMD 2020) Institution of Engineering and Technology . https://doi.org/10.1049/icp.2021.1060

\section{Published in:}

The 10th International Conference on Power Electronics, Machines and Drives (PEMD 2020)

\section{Citing this paper}

Please note that where the full-text provided on Manchester Research Explorer is the Author Accepted Manuscript or Proof version this may differ from the final Published version. If citing, it is advised that you check and use the publisher's definitive version.

\section{General rights}

Copyright and moral rights for the publications made accessible in the Research Explorer are retained by the authors and/or other copyright owners and it is a condition of accessing publications that users recognise and abide by the legal requirements associated with these rights.

\section{Takedown policy}

If you believe that this document breaches copyright please refer to the University of Manchester's Takedown Procedures [http://man.ac.uk/04Y6Bo] or contact uml.scholarlycommunications@manchester.ac.uk providing relevant details, so we can investigate your claim.

\section{OPEN ACCESS}


This paper is a postprint of a paper submitted to and accepted for publication at The 10th International Conference on Power Electronics, Machines and Drives (PEMD 2020) and is subject to Institution of Engineering and Technology Copyright. The copy of record is available at the IET Digital Library.

\title{
A PSCAD PROCESSOR-IN-THE-LOOP SYSTEM FOR HARDWARE EVALUATION OF POWER CONVERTER CONTROL ALGORITHMS Jack Andrews ${ }^{*}$, Peter R Green ${ }^{1}$, Mike Barnes ${ }^{1}$
}

\author{
${ }^{1}$ Department of Electrical and Electronic Engineering, The University of Manchester, Manchester, UK \\ *jack.andrews@manchester.ac.uk
}

Keywords: PSCAD, REAL-TIME SIMULATION, POWER SYSTEM SIMULATION, HVDC

\begin{abstract}
Hardware implementation of controllers for modular multilevel converter voltage source converters is a non-trivial task, which requires a thorough evaluation of the real-world performance of control hardware. This paper presents a new processor-in-theloop system developed at The University of Manchester which enables real-time evaluation of power converter control algorithms implemented on a target hardware platform from within a PSCAD/EMTDC simulation. The developed system has been used to measure the execution times of sorting algorithms implemented within the capacitor balancing control loop of an MMC. The system is a low-cost alternative to commercial real-time hardware-in-the-loop systems, and can be used to bridge the gap between offline simulation and final controller implementation in hardware. The developed system can be extended to support any control algorithm of interest, implemented on a wide range of target hardware platforms which may include: embedded microcontrollers, digital signal processors, and field-programmable gate arrays. Applications include control systems for power electronics connected to power systems including HVDC, renewable energy and smart grids.
\end{abstract}

\section{Introduction}

Modular multilevel converters (MMC) are increasingly being deployed in high voltage direct current (HVDC) transmission applications. When compared to two- and three-level voltagesource converters (VSC), MMCs possess several advantages including: reduced ac output harmonic content, a smaller converter footprint, and reduced switching frequency $[1,2]$. These advantages make MMCs ideal for HVDC applications, such as the connection of offshore wind farms to onshore grids, and sub-sea interconnectors. MMCs, or converters based on variants of the MMC topology have been commercialised by ABB, Siemens, GE Grid Solutions and others [3-5].

A common feature across all MMC topologies is their modular construction from multiple, identical building blocks known as 'submodules'(SM), which are connected in series across the dc link as shown in Fig. 1a. Each phase comprises an upper and lower arm, with the ac output voltage, $v_{a}$, taken from the mid-point of the upper and lower arms. The simplest SM topology is the half-bridge SM (HB-SM), which consists of two switches, $S_{1}$ and $S_{2}$, diodes, and a capacitor, $C_{S M}$, as shown in Fig. 1b. The voltage across the SM, $v_{S M}$, can be switched between $O V$ and $v_{c a p}$ by changing the states of the switches; these are summarised in Table 1. The ac output voltage is controlled by inserting and bypassing SMs in sequence to generate a multi-level stepped output waveform which closely approximates the reference sinusoid.

Industrial scale HVDC MMCs can output several hundred discrete voltage levels, such as the 401-level INELFE link [6] or the 201-level Trans Bay Cable system [7]. The number of SMs in an arm is related to the number of levels by $N-1$, therefore, taking the INELFE link as an example, each arm comprises $400 \mathrm{SMs}$. It is immediately evident that control of an MMC is inherently more complex than previous VSC topologies since each submodule must be controlled in coordination with the other submodules in a phase to achieve several control objectives simultaneously. These are: ac output current control, circulating current suppression, and submodule capacitor balancing control (CBC).
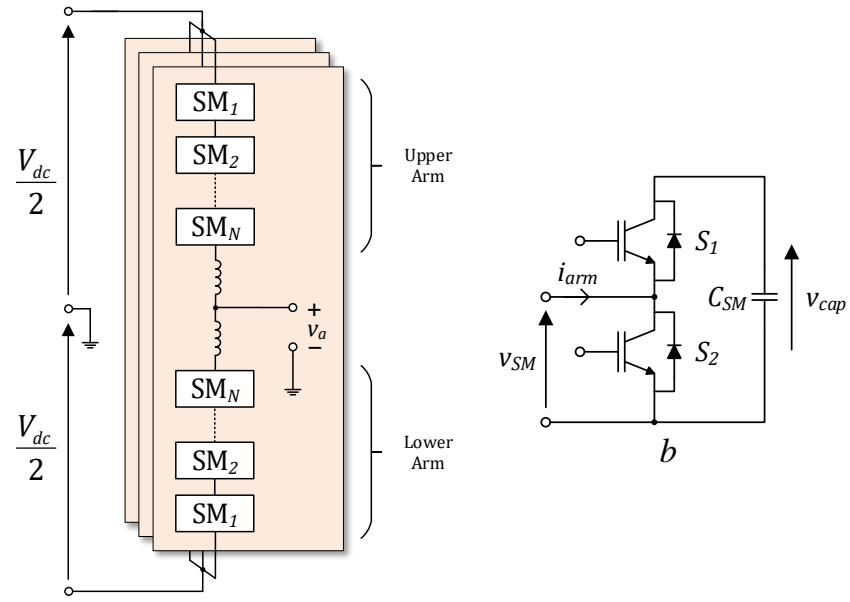

Fig. 1 (a) single phase of an MMC, (b) a half-bridge SM

Table 1 List of states for a HB-SM

\begin{tabular}{llcl}
\hline$S_{1}$ & $S_{2}$ & $v_{S M}$ & State Name \\
\hline OFF & OFF & - & Blocked \\
ON & OFF & $v_{c a p}$ & Inserted \\
OFF & ON & OV & Bypassed \\
ON & ON & - & Not Permitted \\
\hline
\end{tabular}




\section{Capacitor Balancing Control}

The CBC loop in an MMC works to maintain the voltage across each SM capacitor as close as possible to its target value; this prevents damage to the capacitor or SM switches due to overvoltage, and also serves to balance the energy stored in the arm [8]. Under normal operating conditions, the voltage across a single SM capacitor exhibits a ripple due to the energy exchange between phases and the power flow from dc to ac terminals, or vice-versa. When a SM is in the inserted state, $C_{S M}$ charges or discharges dependent upon the direction of the current through the arm, $i_{\text {arm }}$, as shown in Table 2.

Table 2 Arm current direction vs. SM capacitor state

\begin{tabular}{lcll}
\hline State Name & $i_{\text {arm }}$ & \multicolumn{1}{c}{$C_{S M}$} & $v_{\text {cap }}$ \\
\hline Inserted & $>0$ & Charge & $\uparrow$ \\
Inserted & $<0$ & Discharge & $\downarrow$ \\
Bypassed & N/A & Stable & $\rightarrow$ \\
Blocked & N/A & Stable & $\rightarrow$ \\
\hline
\end{tabular}

Implementing the $\mathrm{CBC}$ loop in software running on hardware operating in real-time presents a particular challenge, since almost all CBC methods require the generation of a sorted list of submodule capacitor voltages [9-11]. The CBC loop uses this list to select which SMs to insert or bypass to achieve the arm voltage set point whilst ensuring that the capacitor voltages are balanced. The sorting algorithm chosen for use in the CBC loop is sometimes discussed in literature on MMC control [12, 13], however the algorithms are often overlooked on the assumption that advances in control hardware processing capability have rendered sorting negligible in execution time. Whilst this is a reasonable assumption for reduced-scale prototype MMCs with relatively few submodules, the execution time of widely-implemented sorting algorithms such as bubble sort, and merge sort scales with a power law as the number of values to be sorted is increased [14]. As a result, sorting algorithm execution time rapidly becomes an issue for industrial scale MMCs with several hundred SMs, such as those discussed previously. In addition, the CBC loop is typically triggered at a high frequency in order to produce an effective switching frequency in the low kilohertz range, further increasing the hardware requirements of any prospective target control hardware.

\section{Controller Development Process}

Given these requirements, it is necessary to ensure that the target hardware platform is capable of executing the sorting algorithm within the time constraints of the CBC loop in realtime. This process is the same for any controller and involves several steps before the controller can be integrated into the final system. These steps can be summarised as follows:

\subsection{Offline Simulation}

The converter and its controller are designed and simulated in an offline simulator such as PSCAD/EMTDC or MATLAB/Simulink. This allows initial verification and tuning of the controller.

\subsection{Translation to Target Hardware Platform}

A suitable hardware platform with sufficient resources such as processor speed, memory and input-outputs (IO) is then chosen to implement the controller. This may be an embedded microcontroller, digital signal processor (DSP) or a fieldprogrammable gate array (FPGA). The controller design is translated from the offline simulator into platform-specific code which executes on the target hardware. This translation process can introduce errors due to hardware effects such as: calculation delays, differences in numeric representation (e.g. floating- vs. fixed-point), and digitisation errors. These effects are not normally modelled by the offline simulator, yet can cause the controller operate incorrectly, therefore it is necessary to validate the hardware realisation of the controller.

\subsection{Processor-in-the-loop (PIL) Testing}

In this step, the controller executes on the target hardware platform, whilst the converter is simulated in the offline simulator. The simulator and controller hardware transfer control inputs and outputs over a communications link at each time step. The controller hardware executes in pseudo-realtime, in that, once the control inputs are received from the offline simulator, they are processed by hardware without interruption by the offline simulator. The accuracy of the converter model is maintained, since the offline simulator still runs in simulated time allowing a small time step to be used.

\subsection{Real-Time Hardware-in-the-loop (RT-HIL) Testing}

In this step, the converter model is ported to a RT-HIL system which is interfaced to the controller hardware using a combination of analogue and digital input-outputs (IO). Both the converter model and controller operate in real-time. A RT-HIL system requires a significant amount of processing power to execute the converter model in real time with a small time step. Accordingly, commercial RT-HIL systems are expensive and rely upon proprietary hardware [15]. Alternative RT-HIL systems have been developed in academia which utilise standard hardware and software to perform the processing [16]. This reduces the cost of the system at the expense of a reduction in processing power, limiting the size of the converter which can be simulated.

A PIL-based evaluation of the controller can be used as a lowcost intermediate step towards implementing a controller in hardware operating in real-time. Using this method it is possible to obtain an accurate estimate of control algorithm execution time, debug implementation errors, and determine processing resource usage.

\section{Existing PSCAD/EMTDC PIL Systems}

Processor-in-the-loop implementations in PSCAD/EMTDC have received limited attention in the literature to date. The authors in [17] developed a PIL system which interfaces PSCAD/EMTDC with a National Instruments (NI) cRIO controller using a text file shared between both platforms. This is implemented as a custom component in PSCAD, which also forces the simulation to run as close as possible to real-time. The system is used to evaluate a hysteresis controller for controlling two bus voltages in a distribution system feeder. 


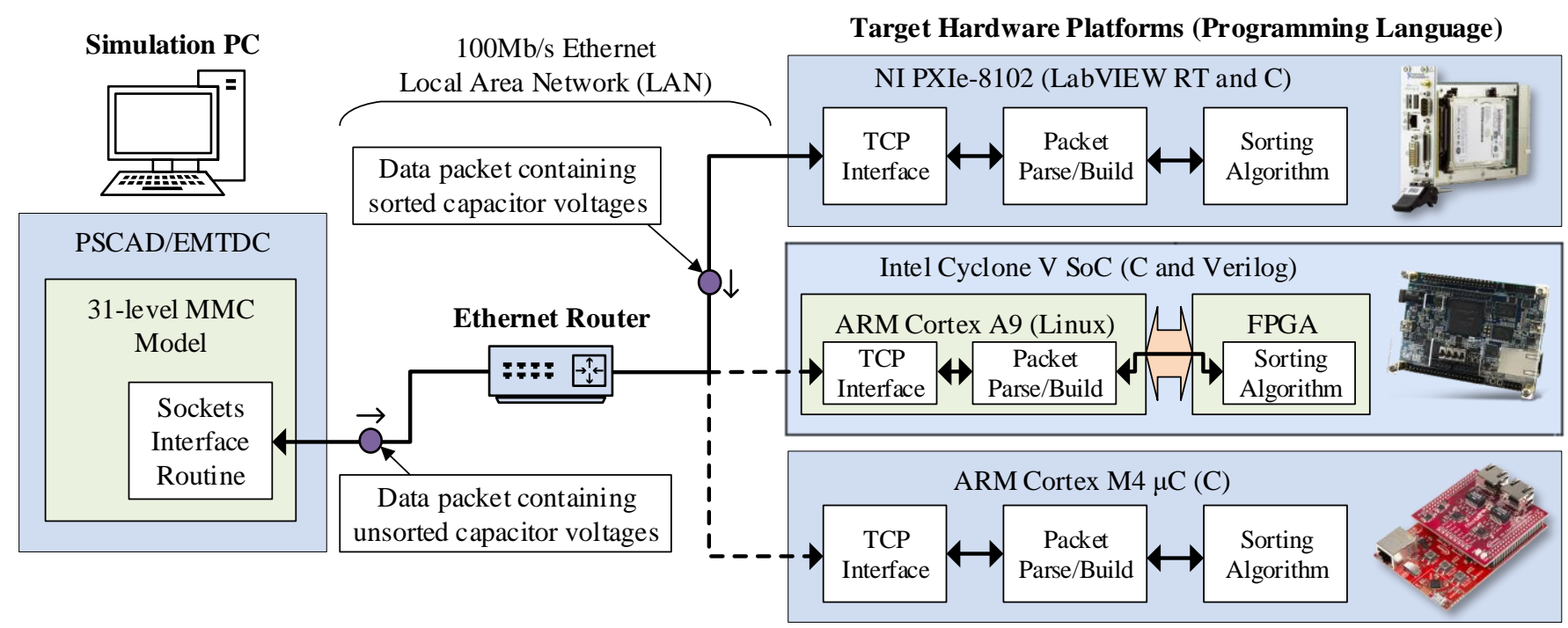

Fig. 2 PSCAD/EMTDC processor-in-the-loop system diagram

The application scope of this PIL approach is limited, since it requires that the target control hardware platform is capable of reading and writing a text file residing in a location accessible to both PSCAD/EMTDC and the controller hardware. Embedded microcontroller platforms and other industriallyrepresentative control platforms typically do not support this type of interface however.

An alternative PIL implementation is presented in [18], in which a PSCAD custom component is developed to read and write from registers in a Renesas microcontroller over an RS-232 serial interface. The system is used to evaluate a dc link and ac bus voltage controller for a STATCOM. The system is then modified in [19] to support a Texas Instruments TMS-series DSP as the target control hardware, and is used to evaluate a controller for a unified power quality conditioner. The PIL system developed by the authors in $[18,19]$ has the potential to support a wider range of target control hardware platforms, however the PSCAD custom component developed is specific to the chosen microcontroller architectures.

The PIL implementation developed by the authors in [20] also utilises an RS-232 serial interface for communication, however the communication protocol is generic and not microcontroller-specific. This in theory allows any target hardware platform with a serial interface to be evaluated from within PSCAD/EMTDC, although this approach is limited by the maximum data rates possible using the RS-232 protocol, which will increase the simulation execution time. The system developed in [20] is used to evaluate a controller for a 3-phase ac motor.

\section{PSCAD/EMTDC PIL System}

The PIL system developed in this work comprises three main pieces of hardware: a PC running PSCAD/EMTDC, a network router, and the target control hardware platform. A model of a 31-level three-phase MMC is implemented in PSCAD/EMTDC and simulates the dynamics of the converter and its submodules, such that the submodule capacitor voltages can be extracted for processing by the sorting algorithm of interest. An Ethernet router is used to establish a communication link between the simulation PC and the target hardware platform over a local area network (LAN). An overview of the system architecture is shown in Fig. 2.

\subsection{Target Hardware Platforms}

In this work, three target hardware platforms have been used with the PIL system to evaluate the sorting algorithms of interest; these are outlined below:

- NI PXIe-8102 controller running a real-time operating system (RTOS).

- Intel Cyclone V system-on-chip (SoC) which integrates a dual-core ARM Cortex A9 hard processor system (HPS) and Intel Cyclone V FPGA in a single package, mounted on a Terasic DE0-Nano development board.

- ARM Cortex M4 embedded microcontroller ( $\mu \mathrm{C})$, mounted on an Infineon XMC4800 Relax development board.

These platforms were chosen since they are representative of the control hardware platforms typically used in industry. In particular, the SoC-FPGA platform was selected for evaluation since industrial controllers typically use FPGAs to implement high bandwidth, complex control loops such as CBC.

\subsection{Communication Interface}

Communication between the PSCAD/EMTDC simulation and the target hardware platform is implemented using a network 'sockets' interface. Sockets are an IEEE standard computer programming abstraction which is available on almost all operating systems (OS), including Microsoft Windows and Linux. The sockets interface allows transmission of raw bytes between two devices, one operating as a 'server' and the other as a 'client'. The client and server sockets may exist on the same device, or on two different devices connected over a LAN, as is the case in this work. In this case, PSCAD/EMTDC behaves as the client and the target hardware platform as the server. 
Behind the sockets abstraction layer, the transmission control protocol (TCP) is used to transfer data packets over the LAN. Many embedded microcontrollers now have libraries available to enable communication using TCP over a LAN, allowing them to be used with this PIL system.

\subsection{Interface Routines}

The sockets interface routines on both the simulation PC and target hardware platform serve to initiate the connection between the two machines during the first simulation time step, and to build, send, receive and parse data packets containing SM capacitor voltages for sorting during subsequent time steps in the simulation run.

PSCAD Interface: In PSCAD a custom component has been created which implements the CBC loop and the PIL interface. The PIL interface routines are written in the $\mathrm{C}$ programming language; these functions are called from within the FORTRAN code of the CBC loop during a simulation run. The custom component also allows configuration of the connection to the target hardware platform and logging for debugging purposes.

Target Hardware Platform Interface: The target hardware platform interface is split into two separate components:

- Platform-specific code which implements communication with the simulation PC.

- Platform-independent libraries which handle building and parsing of data packets containing capacitor voltages.

The output of the platform-specific communication code is a buffer containing the data packet received from the simulation PC. This buffer is passed to the library code which then parses the packet and places the data into an array for manipulation by the sorting algorithm. The library code is written in $\mathrm{C}$ to enable portability across a range of target hardware platforms.

\subsection{System Operation and Synchronisation}

The PIL system operates according to the sequence diagram shown in Fig. 3. Prior to commencement of the simulation run, the target hardware platform is powered on and waits in a 'listen' state for an incoming connection from the simulation PC. The PSCAD/EMTDC simulation is then started, and in the first time step a function is called to 'open' a socket connection to the listening target hardware platform, which then 'accepts' the incoming connection.

At each discrete time step, $n \Delta t$, the PSCAD/EMTDC solver processes the converter dynamics and calculates the submodule capacitor voltages for the current time step, which are then passed to the CBC controller PSCAD custom component. The simulation is paused at this point, and waits for the PIL system to finish processing as follows: the PSCAD interface routine builds a packet containing the unsorted capacitor voltages from the current time step, and sends the packet to the target hardware platform across the sockets connection between the two machines. Once the packet is received, the capacitor voltages are parsed and the sorting algorithm is executed in real time by the target hardware platform. Upon completion of the sorting algorithm, the sorted values are assembled into a packet and sent back to the PSCAD/EMTDC simulation. Once the sorted values have been received and parsed, the simulation resumes processing the converter dynamics for the current time step, then advances to the next time step. In the final time step, the simulation calls a function to close the connection with the target hardware platform.

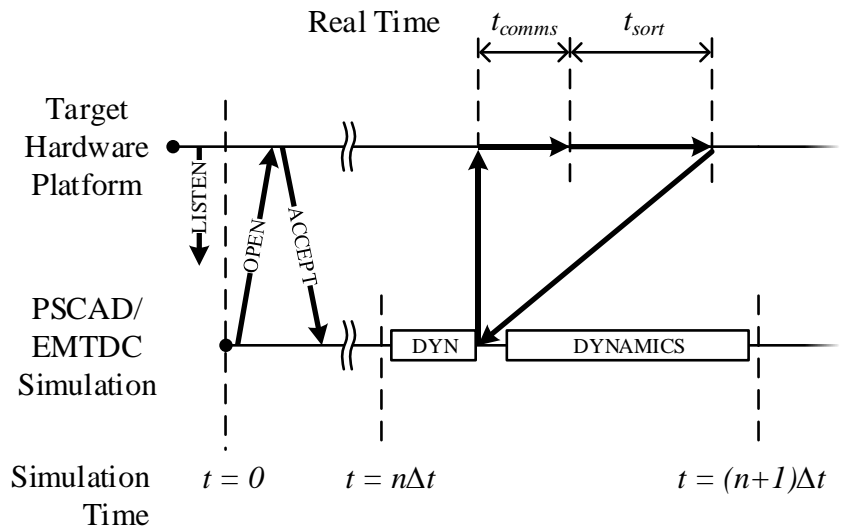

Fig. 3 PIL system sequence diagram

Since the target hardware platform executes the sorting algorithm in real time, the execution time of the algorithm, $t_{\text {sort }}$, can be accurately measured using a timer peripheral or by toggling an IO pin connected to an oscilloscope. The processing delays introduced by both the PSCAD and target hardware platform interface routines, and the communication delays across the LAN are represented by a single delay, $t_{\text {comms }}$.

\section{PSCAD/EMTDC PIL System Performance}

The PIL system developed in this work has been evaluated to determine the additional processing and communication delays introduced into a PSCAD/EMTDC simulation when the PIL interface is enabled. The length of these delays is influenced by two main factors:

- Data packet length: the quantity and type of values being transmitted and received by PSCAD/EMTDC at each simulation time step.

- Target hardware platform processing speed: this affects the execution time of both the control algorithm and the platform-independent library code which parses and builds the data packets.

Besides these factors, the communication delay is also affected by elements which are outside direct control of the user of the PIL system. These include delays in the router as the data packet is processed, and in the OS networking software running on the simulation PC due to network traffic from other applications. Delays due to these factors exhibit themselves as a variation in $t_{\text {comms }}$ even when the target hardware platform and data packet length are kept constant. These delays are difficult to quantify and will not be discussed independently here. 


\subsection{Round-trip Time vs. Data Packet Length}

To measure the communication and data packet processing delay, $t_{\text {comms }}$, a loop-back test was performed on each target hardware platform. For this test, the outputs of the data packet parse routine were fed directly into the inputs of the data packet build routine, such that the delay due to $t_{\text {sort }}$ (or indeed any other control algorithm) was eliminated.

As stated previously, the data packet length is dictated by the number and type of values to be transmitted. PSCAD/EMTDC has three main data types: logical, integer, and real, which the PIL PSCAD custom component also supports. When encoded in a data packet, these occupy 2,9 , and 26 bytes respectively per value, in addition to 55 bytes of header and trailer data. The data packet length, $L_{p k t}$, can be calculated as shown in equation (1).

$$
L_{p k t}=55+2 \cdot n_{\text {logical }}+9 \cdot n_{\text {integer }}+26 \cdot n_{\text {real }}
$$

A range of data packet lengths were tested from the shortest possible length of 57 bytes, representing the transmission of a single logical value, up to 499 bytes, representing the transmission of 12 logical, 12 integer and 12 real values. The loop-back test was repeated 10000 times for each target hardware platform and data packet length combination, and the mean value of $t_{\text {comms }}$ calculated. The results for each target hardware platform are shown in Fig. 4.

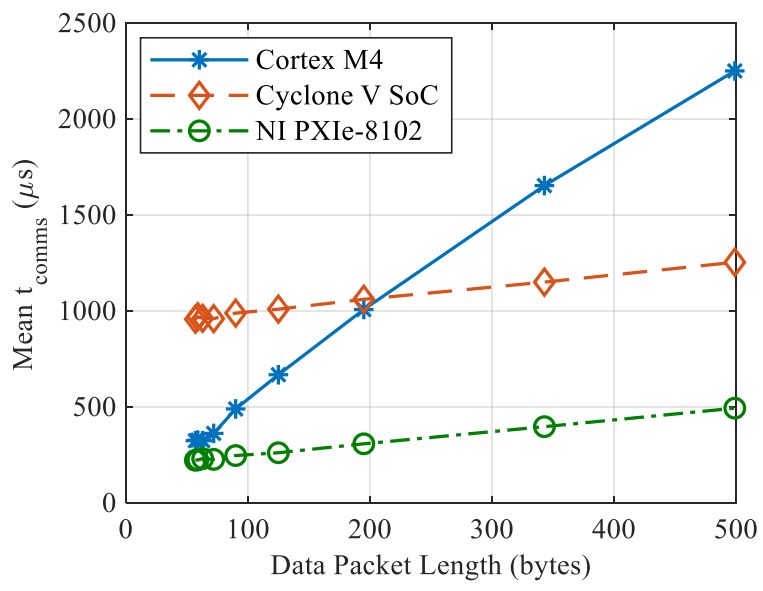

Fig. 4 Data packet length vs. $t_{\text {comms }}$ for Cortex M4 (solid), Cyclone V SoC (dashed) and NI PXIe-8102 (dash-dot) hardware platforms

As can be seen from the results in Fig. 4, a linear relationship exists between $t_{\text {comms }}$ and data packet length for all platforms. The NI PXIe-8102 platform has the lowest values of $t_{\text {comms }}$ across all data packet lengths, due to the fact its processor has the highest clock frequency $(1.9 \mathrm{GHz})$ out of all the platforms. The Cyclone V SoC exhibits a very similar gradient to the NI platform, except with a greater offset; this is due the ARM Cortex A9 processor having a lower clock frequency of $925 \mathrm{MHz}$. Whilst the Cortex M4 platform exhibits a $t_{\text {comms }}$ delay only approximately $100 \mu$ s greater than that of the NI platform when the data packet length is less than 75 bytes, the delay increases more rapidly compared to the two other platforms. This is almost certainly due to the fact that the
Cortex M4 has only a single processor core, whilst both the Cyclone V SoC and NI platforms utilise a dual-core processor. In a single core processor, the networking (TCP) software and data packet processing routines must execute sequentially, whereas in a dual-core processor, the networking software can execute in parallel to the data packet processing routines in a different processor core, reducing the delay.

\subsection{Simulation Runtime vs. Target Hardware Platform}

To evaluate the effects of the PIL system upon the total simulation runtime, each target hardware platform was configured to perform sorting of SM capacitor voltages within the CBC loop of an MMC modelled in PSCAD/EMTDC. The same 'bubble sort' algorithm was used on all of the platforms and was chosen for its ease of implementation. The execution time of the sorting algorithm was also measured in this test. For comparison purposes, simulation runtime was also measured with bubble sort implemented natively in FORTRAN code in PSCAD/EMTDC, and in MATLAB using the native MATLAB interface. The MMC model employed for this test is a 31-level detailed equivalent model. The converter was set to import $800 \mathrm{MW}$ and $600 \mathrm{MVAr}$ of power, and a $\mathrm{CBC}$ loop trigger frequency of $1.5 \mathrm{kHz}$ was used. The simulation run duration was 3 seconds with a time step of $20 \mu \mathrm{s}$. The results for each sorting algorithm target hardware platform are shown in Table 3.

Table 3 Simulation runtime, $t_{\text {sort }}$ and $t_{\text {comms }}$ results for different target hardware platforms

$\begin{array}{cccc}\text { Sorting Algorithm } & \text { Total } & \text { Mean } & \text { Mean } \\ \text { Target Hardware } & \text { Simulation } & t_{\text {sort }} & t_{\text {comms }} \\ \text { Platform } & \text { Runtime } & (\mu \mathrm{s}) & (\mu \mathrm{s})\end{array}$

(s)

\begin{tabular}{lllr}
\hline Baseline (PIL disabled) & 18.72 & 3.54 & \multicolumn{1}{c}{-} \\
NI PXIe-8102 & 25.31 & 29.5 & 952 \\
Cyclone V SoC & 27.46 & 14.5 & 1700 \\
Cortex M4 & 360.5 & 179 & 47600 \\
MATLAB interface & 58.01 & 69.1 & 3610 \\
\hline
\end{tabular}

As expected, the total simulation runtime is fastest when the PIL system is disabled, since the simulation operates in isolation with no communication overhead. For each of the remaining platforms, the total simulation runtime is highly dependent upon $t_{\text {comms }}$ for each platform, which is the dominant cause of delay in the PIL subsystem. This is especially true for the Cortex M4 $\mu \mathrm{C}$. Communication delay also dominates the total delay when using the MATLAB interface, despite the fact that MATLAB runs alongside the simulation on the same PC.

\section{Conclusion}

A new processor-in-the-loop system has been presented, which enables the evaluation of power converter control algorithms in implemented real-time hardware from within a PSCAD/EMTDC simulation. The performance of the PIL system has been evaluated on three industrially-representative target hardware platforms to determine the delays which can be expected when using the system. With the exception of the 
Cortex M4 platform, using the PIL system does not cause an unacceptable increase in total simulation runtime. The system developed in this work can be used as a low-cost, intermediate step in the evaluation of control algorithms implemented in software running in real-time on hardware, instead of, or before porting to a RT-HIL system.

Further development of the functionality of the system is possible. This includes providing the option to use different communication interfaces, such as RS-232, to enable a wider range of target hardware platforms to be used with the system. Should there be sufficient interest, the authors will consider publishing the PSCAD custom component for the PIL system and developing the system further.

\section{Acknowledgements}

The authors would like to thank Antony Beddard for the use of the detailed equivalent model of the 31-level MMC developed during his $\mathrm{PhD}$. This work was supported by the HOME Offshore Project through Engineering and Physical Sciences Research Council (EPSRC) grant EP/P009743/1.

\section{References}

[1] Oates, C.: 'Modular Multilevel Converter Design for VSC HVDC Applications', IEEE Trans. Emerg. Sel. Topics Power Electron., 2015, 3, (2), pp. 505-515

[2] Barnes, M., Beddard, A.: 'Voltage Source Converter HVDC Links - The State of the Art and Issues Going Forward', Energy Procedia, 2012, 24, pp. 108-122

[3] Jacobson, B., Karlsson, P., Asplund, G., et al.: 'VSCHVDC Transmission with Cascaded Two-Level Converters'. Proc. CIGRE Session 2010, Paris, France, August 2010, pp. 17

[4] Friedrich, K.: 'Modern HVDC PLUS application of VSC in Modular Multilevel Converter topology'. Proc. 2010 IEEE Int. Symposium on Industrial Electronics, Bari, Italy, July 2010, pp. 3807-3810

[5] Chivite-Zabalza, J., Perrier, J., Boden, M., et al.: 'Development of a Full-Bridge Sub-Module for HVDC and STATCOM Markets'. Proc. Int. Exhibition and Conf. Power Electronics, Intelligent Motion, Renewable Energy and Energy Management, Nuremberg, Germany, May 2017, pp. 17

[6] Francos, P.L., Verdugo, S.S., Alvarez, H.F., et al.: 'INELFE Europe's first integrated onshore HVDC interconnection'. Proc. 2012 IEEE Power and Energy Society General Meeting, San Diego, USA, July 2012, pp. 1-8

[7] Westerweller, T., Friedrich, K., Armonies, U., et al.: 'Trans bay cable - world's first HVDC system using multilevel voltage-sourced converter'. Proc. CIGRE Session 2010, Paris, France, August 2010, pp. 1-7

[8] Lizana, R., Perez, M.A., Bernet, S., et al.: 'Control of Arm Capacitor Voltages in Modular Multilevel Converters', IEEE Trans. Power Electron., 2016, 31, (2), pp. 1774-1784
[9] Meshram, P.M., Borghate, V.B.: 'A simplified nearest level control (NLC) voltage balancing method for modular multilevel converter (MMC)', IEEE Trans. Power Electron., 2014, 30, (1), pp. 450-462

[10] Hassanpoor, A., Ilves, K., Norrga, S., et al.: 'Toleranceband modulation methods for modular multilevel converters'. Proc. 15th European Conf. Power Electronics and Applications, Lille, France, September 2013, pp. 1-10

[11] Tu, Q., Xu, Z., Xu, L.: 'Reduced Switching-Frequency Modulation and Circulating Current Suppression for Modular Multilevel Converters', IEEE Trans. Power Del., 2011, 26, (3), pp. 2009-2017

[12] Ricco, M., Mathe, L., Teodorescu, R.: 'New MMC capacitor voltage balancing using sorting-less strategy in nearest level control'. Proc. IEEE Energy Conversion Congress and Exposition, Milwaukee, USA, September 2016, pp. 1-8

[13] Zhao, F., Xiao, G., Song, Z., et al.: 'Insertion sort correction of two-way merge sort algorithm for balancing capacitor voltages in MMC with reduced computational load'. Proc. IEEE 8th Int. Power Electronics and Motion Control Conf., Hefei, China, May 2016, pp. 748-753

[14] Cormen, T.H., Leiserson, C.E., Rivest, R.L., et al.: 'Introduction to Algorithms' (The MIT Press, 1990, 3rd edn. 2009)

[15] Faruque, M.D.O., Strasser, T., Lauss, G., et al.: 'RealTime Simulation Technologies for Power Systems Design, Testing, and Analysis', IEEE Power Energy Technol. Syst. J., 2015, 2, (2), pp. 63-73

[16] Lu, B., Wu, X., Monti, A.: 'Implementation of a low-cost real-time virtue test bed for hardware-in-the-loop testing'. Proc. 31st Annu. Conf. of IEEE Industrial Electronics Society, Raleigh, USA, November 2005, pp. 239-244

[17] Ziaeinejad, S., Mehrizi-Sani, A.: 'Software-based hardware-in-the-loop real-time simulation of distribution systems'. Proc. 2016 IEEE Power and Energy Society General Meeting, Boston, USA, July 2016, pp. 1-5

[18] Rolim, L.G.B., Miranda, U.A., França, B.W., et al.: 'Hardware-in-the-loop evaluation of DSP-controlled converters'. Proc. Brazilian Power Electronics Conf., BonitoMato Grosso do Sul, Brazil, September 2009, pp. 805-809

[19] França, B.W., Rolim, L.G.B., Aredes, M.: 'Frequency switching analysis of an iUPQC with hardware-in-the-loop development tool'. Proc. 2011 14th European Conf. on Power Electronics and Applications, Birmingham, UK, August 2011, pp. 1-6

[20] Chongva, G., Filizadeh, S.: 'Non-real-time hardware-inloop electromagnetic transient simulation of microcontrollerbased power electronic control systems'. Proc. 2013 IEEE Power and Energy Society General Meeting, Vancouver, Canada, July 2013, pp. 1-5 